\title{
Oral Administration Following Subcutaneous Administration of FCV Vaccines Enhances Vaccine Efficacy against Challenge of a Highly Virulent Vs Feline Calicivirus
}

\author{
Sing Rong ${ }^{*}$, Kim Floyd-Hawkins ${ }^{2}$, Vicki King ${ }^{2}$ \\ ${ }^{1}$ Global Biologics Research, Zoetis-Vet Med R \& D, Kalamazoo, USA \\ ${ }^{2}$ Global Development \& Operation, Zoetis-Vet Med R \& D, Kalamazoo, USA \\ Email: sing.rong@zoetis.com
}

Received 6 December 2013; revised 6 January 2014; accepted 13 January 2014

Copyright (C) 2014 by authors and Scientific Research Publishing Inc.

This work is licensed under the Creative Commons Attribution International License (CC BY). http://creativecommons.org/licenses/by/4.0/

\section{(c) (i) Open Access}

\section{Abstract}

Feline calicivirus (FCV) is a common cause of upper respiratory and oral disease in cats. Highly virulent systemic strains of FCV (vs FCV) have been described. These vs FCV isolates cause characteristic edema, cutaneous ulcers and other clinical signs typically associated with FCV infection. Vs FCV isolates also cause high mortality even in previously vaccinated cats. We reported previously that the FCV serum cross-neutralization profile of cat serum generated using the oralnasal route of administration is broader than with subcutaneous administration (SC), as measured with a 26-FCV viral panel (Rong et al., Virus Research 122:95-108, 2006). In this report, we tested the in vivo efficacy of the FCV vaccine, in a 4-way (FCV-FHV-FPV-FCp) format, by using a highly virulent vs FCV33585 as the challenge virus. Vaccines were administered as 2-dose subcutaneouly (SC/SC), or subcutaneously followed by orally (SC/Oral). The mortality induced by vs FCV-33585 in unvaccinated control cats was $78 \%$ ( 7 out of 9 cats). The mortality decreased to $44 \%$ (4 out of 9 cats) with cats vaccinated with the 4-way vaccine given SC/SC. However, when this vaccine was given SC/Oral, the mortality decreased to $10 \%$ ( 1 out of 10 cats). The clinical scores, calculated based on frequency and severity of various clinical signs, correlated with mortality data. These results demonstrated that oral administration of FCV vaccines, as the second dose following the first dose of subcutaneious administration, ehances FCV efficacy against challenge of a highly virulent vs FCV. We propose that not only oral vaccination offers convenience and needle-free inoculation, it also enhances FCV vaccine efficacy.

*Corresponding author.

How to cite this paper: Rong, S., et al. (2014) Oral Administration Following Subcutaneous Administration of FCV Vaccines Enhances Vaccine Efficacy against Challenge of a Highly Virulent Vs Feline Calicivirus. World Journal of Vaccines, 4, 81-87. 


\section{Keywords}

\section{Positive-Strand RNA, FCV, VS FCV, Calicivirus, Subcutaneous Administration, Oral/Oronasal Administration, Serum-Virus Neutralization, Vaccine Efficacy}

\section{Introduction}

Caliciviruses are a family of small viruses that possess a positive-strand, non-segmented, polyadenylated RNA genome of about $7.5 \mathrm{~kb}$. Members of this family include feline calicivirus (FCV), rabbit hemorrhagic disease virus (RHDV), San Miguel sea lion virus (SMSV), vesicular exanthema virus of swine (VEV) and many human caliciviruses [1] [2]. Entry of FCV into cells was shown to be dependent on clathrin-mediated endocytosis and acidification in endosomes [3]. Junctional adhesion molecule 1 (JAM-1) is a functional receptor for FCV [4] [5].

The signs of illness produced by FCV infections in the cat vary markedly, depending on the strain involved, the severity of the exposure and the resistance of the host [2]. Strains of low virulence produce not only moderate pyrexia but also can produce ulcers of the tongue, hard palate and nose. Strains of high virulence (virulent systemic strains, vs FCV) produce pyrexia, anorexia, depression, transient lameness, dyspnea or polypnea and frequently death, affecting adults cats as well as kittens [6]-[14]. In addition to pneumonic signs produced by vsFCV isolates, ulcers of the tongue, hard palate and nose may also occur. US, UK, as well as France and Germany, all reported vs FCV outbreaks [15] [16].

Despite the availability of vaccines for immunizing cats against FCV, none of the present vaccines have been shown to be completely protective against all strains of FCV due to continously emergening new field isolates [17]-[20]. Outbreaks of vs FCV have occurred within well-vaccinated cat populations [21] [22]. Therefore, there is a need for an efficacious and safe vaccine that affords protection to cats against both the highly virulent vs FCV, and also the newly emergent field strains. We have reported previously that the serum cross-neutralization profile of FCV F9 was broader when the vaccine virus was inoculated oronasally when compared with subcutaneous inoculation (SC) [14]. In this study, we tested feline 4-way (FCV F9-FHV-FPV-FCp) vaccines, administrated with 2-dose subcutaneouly (SC/SC), or subcutaneously followed by orally (SC/Oral). Efficacy, as measured by mortality and clinical scores induced by a vs FCV-33585 challenge, was measured and the serum crossneutralization profile was assessed for each treatment group.

\section{Materials and Methods}

\subsection{Cells and Viruses}

CRFK (cat kidney cells, ATCC CCL-94) cells were used for routine culture of FCV.

The growth medium was DMEM (Invitrogen) supplemented with antibiotics and 3\% - 10\% gamma-irradiated fetal bovine serum (Cambrex Bio Science Rockland).

Vs FCV-33585 was described in Rong et al. [14]. FCV-F9 virus used in the study is a vaccine strain. Twentysix FCV strains that were used in the serum cross-neutralization assay are listed in the table below. They were selected based on their geographic representation, as well as their unique DNA/protein sequences in the viral capsid hypervirable region, amino acid 424 - 473 [14].

\begin{tabular}{|c|c|c|c|c|c|c|c|c|}
\hline \multicolumn{9}{|c|}{ FCV strains in serum cross-neutralization viral panel: } \\
\hline Strain & Year & Location & Strain & Year & Location & Strain & Year & Location \\
\hline $12217-02$ & 2002 & NY & $84883-02$ & 2002 & NY & 88287 & 2000 & PA \\
\hline 19306 & 2003 & FL & F9 & 1960 & Not Known & 89391 & 2000 & PA \\
\hline $26391-4$ & 2003 & FL & $\mathrm{J}-1$ & 1984 & $\mathrm{CT}$ & $101920-1$ & 2002 & NY \\
\hline $27086-2$ & 2003 & FL & $\mathrm{H}$ & 1990 & $\mathrm{AZ}$ & $17932-17$ & 2003 & RI \\
\hline $32561-1$ & 2003 & IN & 2280 & 1983 & Not Known & $30101-2$ & 2003 & MT \\
\hline $32561-14$ & 2003 & IN & 255NVSL & 1970 & Not Known & 41927-8 & 2003 & $\mathrm{CO}$ \\
\hline $32561-15$ & 2003 & IN & 94580 & 2000 & NY & FCV-21 & 1993 & MI \\
\hline $32561-7$ & 2003 & IN & $100869-1$ & 2000 & Ontario & FCV-49 & 1993 & PA \\
\hline $36069-2$ & 2003 & MT & 33585 & 2000 & MA & & & \\
\hline
\end{tabular}




\subsection{Efficacy Study against Challenge of a Vs Strain FCV-33585 Using 2-Dose Vaccination Regimen}

Domestic shorthair cats, about 8 weeks of age, were randomly assigned to three different treatment groups consisting of a control group, and two vaccine groups using a randomized complete block design. Animals were blocked on date of birth. The experimental 4-way (FCV F9-FHV-FPV-FCp) vaccines contained modified-live FCV F9, feline rhinotracheitis virus (FHV), panleukopenia virus (FPV), and Chlamydia psittaci (FCp). The vaccination regimens included an initial subcutaneous vaccination followed by subcutaneous boosts on day 21 (SC/SC); or an initial subcutaneous vaccination followed by one oral (sublingual) booster immunization on day 21 (SC/Oral). Each cat within the different dosing regimens (10 cats per group) received $1 \mathrm{~mL}$ of experimental reconstituted lyophilized vaccines.

On day 42, all cats were challenged by oranasal route with approximately $1 \mathrm{~mL}$ of virulent systemic FCV33585 (3 log of $\mathrm{TCID}_{50} / \mathrm{mL}$ ). For 14 days post-challenge, all cats were monitored for clinical signs (temperature, conjunctivitis serous discharge, conjunctivitis mucopurulent discharge, rhinitis serous discharge, rhinitis mucopurulent discharge, sneezing, audible rales, coughing open mouth breathing, anorexia, dehydration, one oral ulcer $<4 \mathrm{~mm}$, multiple oral ulcers, oral ulcers $>4 \mathrm{~mm}$, salivating, nonbleeding external ulcer, bleeding external ulcers) of disease for 14 days post-challenge. Cats exhibiting severe clinical signs post-challenge that were consistent with calicivirus pathogenesis were euthanized.

Clinical scoring was done on a "point" system. For each clinical sign, there are "X" points awarded. The following is the abbreviated version:

Conjunctivitis: None $=0$, Serous Discharge $=1$, Mucopurulent Discharge $=2$.

Rhinitis: None $=0$, Serous Discharge $=1$, Mucopurulent Discharge $=2$.

Sneezing: None $=0$, Occasional $=1$, Persistent $=2$.

Dyspnea: None $=0$, Audible Rales $=1$, Coughing $=2$, Open Mouth Breathing $=3$.

Dehydration: None $=0$, Mild $=1$, Severe $=2$.

Oral Ulcers: None $=0$, One $(<4 \mathrm{~mm})=1$, Multiple $(<4 \mathrm{~mm})=2$, Any $>4 \mathrm{~mm}=3$.

Lethargy/Depression: None $=0$, Mild $=1$, Severe $=2$.

Salivating: No $=0$, Yes $=1$.

External Ulcers: None $=0$, Present $=2$.

Swelling/Facial lesions: None $=0$, Mild $=1$, Severe $=2$.

Lameness: None $=0$, Mild $=1$, Severe $=2$.

Anorexia: None $=0$, Mild $=1$, Severe $=2$.

Diarrhea: No $=0$, Yes $=1$.

The cats were evaluated on a daily basis for each of the above. They are assigned "points" daily and given a "total” score for each day. A low score indicates a healthier animal than one with a higher score. All data analysis was preformed with SAS Release 9.1.1. Clinical scores were calculated using a scoring system that had been previously used by the USDA (MVSAM0310.01). The clnical scores were anlayzed using the KruskalWallis Test. Mortality was analyzed using Fisher's Exact Test. Since the animals were housed by treatment group during the vaccination phase of the study only treatment geometric means, minimums and maximums were calcualted for each time point.

All procedures in this study were approved and conducted in compliance with the Animal Welfare Act Regulations (9 CFR Parts 1, 2 and 3) and the "Guide for the Care and Use of Laboratory Animals” (ILAR, 1996), as well as with all internal company policies and guidelines.

\subsection{Serum-Virus Neutralization Analysis against a 26-FCV Panel}

Serum samples were collected from each of the vaccinated cats 2 weeks post the $2^{\text {nd }}$ vaccination and heat treated at $56^{\circ} \mathrm{C}$ for 30 minutes. They were used in the serum neutralization assay against a panel of 26 FCV viral strains as described previously [14].

\subsection{Serology Analysis for FHV, FPV \& FCp}

Serum neutralization titers were determined for FHV, FPV \& FCp components for the vaccine groups following challenge study. Post-vaccination \& pre-challenge serum was used for FPV titer, while post-challenge serum 
was used for FHV \& FCp titers. The titration studies were performed by Benchmark Biolab (Lincoln, Nebraska) in their standard tests.

\section{Results}

\subsection{Serum Neutralization (SN) Titers against FCV F9 Administrated via SC (Subcutaneously) or ON (Oronasal) Routes}

We reported previously that the serum cross-neutralization profile was enhanced when FCV-F9 was vaccinated oronasally (ON) when compared with subcutaneous vaccination (SC) [14]. The data were reformatted and represented in Table 1. Using 23 as the cut-off value for neutralization titers, ON vaccination resulted in $26 \%$ of the 26 FCV-panel members neutralized, while SC vaccination resulted in only $16 \%$ neutralized. There was $163 \%$ enhancement in the cross-neutralization profile when ON was compared with SC, while SC was only $62 \%$ of ON. Using a SN titer cut-off of 15, ON vaccination yielded 32\% of panel members being neutralized, while SC yielded only $17 \%$. There was $188 \%$ enhancement in the cross-neutralization profile when ON was compared with SC, while SC was only 53\% of ON. We hypothesize that the results suggest possible enhanced FCV vaccine efficacy with ON administration when compared with SC administration.

\subsection{Mortality and Clinical Scores for Cats Vaccinated with 2-Dose of 4-Way Vaccines against Vs FCV-33585 Challenge}

FCV F9 vaccine strain is known to have a residual virulence. Therefore, we excluded the possibility of using oral or oronasal routes of administration for the first dose for vaccines containing FCV F9. We reasoned that by vaccination the first dose with SC that was known to be safe, cats would develop enough immunity to make the second oral or oronasal dose safe, despite the residual virulence of FCV F9. The reason we chose oral route, rather than oronasal, as the second dose was based on the assumption that oral administration in cats would be more acceptable and more convenient than oronasal route.

Cats were administered experimental 4-way (FCV F9-FHV-FPV-FCp) vaccines with vaccination regimens either SC/SC or SC/Oral as described in Materials \& Methods. No safety related adverse reactions were observed post the two vaccinations and prior to challenge. We concluded that SC/Oral is as safe as the conventional SC/SC administration.

The challenge strain vs FCV-33585 induced 78\% mortality in control cats. While SC/SC vaccination decreased mortality to $44 \%$, the SC/Oral vaccination regimen reduced mortality further to $10 \%$ (Table 2). The median clinical score for control group is 24 . The SC/SC vaccination decreased the median clinical score to 12, the SC/Oral group reduced the median clinical score to 5.5 (Table 2). These results suggest that SC/Oral administration of FCV vaccine in the 4-way format (FCV F9-FHV-FPV-FCp) improved FCV efficacy against vs FCV33585 challenge, when compared with conventional SC/SC administration.

\subsection{Cross Neutralization Analysis of Sera from Cats Vaccinated with 4-Way Vaccines (FCV F9-FHV-FPV-FCp)}

Serum samples from each cat were collected post second vaccination and before challenge, and used in the serum neutralization assay against each of 26 FCV strains. Serum neutralization data were analyzed with cut-off titers of $>23$ and $>15$ and, an average of $\geq 23$ and $\geq 15$ (Ave). The serum cross neutralization profile of the 4-way vaccine containing FCV-F9 was $41 \%$ for SC/Oral and $40.4 \%$ for SC/SC (Table 3). The second oral dose did not change much the serum cross-neutralization profile. Furthermore, we observed background noise of $12.4 \%$ serum cross-neutralization for the control group (Table 3).

\subsection{SN Titer Analysis of FHV (Post-Challenge Serum), FPV and Chlamydia (FCp) (Pre-Challenge Serum)}

The SN titers of FHV (post-challenge serum), FPV and FCp (pre-challenge serum) were determined using the serum samples from the study. Results show that FPV induced a sufficient immune response for both SC/SC and SC/Oral groups, indicating good FPV efficacy for both groups.

The situations with FHV and FCp are less clear. It is known that serology responses and in vivo protection do 
Table 1. Serum cross-neutralization titers for serum generated SC (subcutaneouly) in comparison with ON (oranasal) for FCV F9 against a 26-FCV viral panel.

\begin{tabular}{ccc}
\hline FCV-F9 & \% Pos. $\left(\mathbf{2 3} \mathbf{3}^{*}\right)$ & \% Pos. $\left(\mathbf{1 5 ^ { * } )}\right.$ \\
\hline SC Inoculaiton & 16 & 17 \\
ON Inoculation & 26 & 32 \\
ON over SC (\%) & 163 & 188 \\
SC over ON (\%) & 62 & 53 \\
\hline
\end{tabular}

${ }^{*}$ Neutralization titer of $\geq 23$ as cut-off value; or neutralization titer of $\geq 15$ as cut-off value.

Table 2. Mortality of cats challenged with vs FCV-33585 following 2-dose vaccination with experimental 3 or 4-way combination vaccines.

\begin{tabular}{cccccccc}
\hline & Vaccination & \multicolumn{2}{c}{ Challenge } & \multicolumn{3}{c}{ Clinical Score } \\
\hline \# Cats & Treatment & Route & \# Cats & Mortality \% & Median & Min. & Max. \\
\hline 10 & Control & SC/SC & 9 & 78 & 24 & 2 & 35 \\
10 & FCV F9 + FHV + FPV + FCp & SC/SC & 9 & 44 & 12 & 3 & 38 \\
10 & FCV F9 + FHV + FPV + FCp & SC/Oral & 10 & 10 & 5.5 & 0 & 30 \\
\hline
\end{tabular}

Table 3. Cross-neutralization profile against 26-FCV viral panel following 2-dose vaccination.

\begin{tabular}{|c|c|c|c|c|c|c|c|}
\hline \multicolumn{3}{|c|}{ Vaccination } & \multicolumn{2}{|c|}{ Serum Collection } & \multicolumn{3}{|c|}{ Cross Neutralization } \\
\hline \# Cats & Treatment & Route & Days & \# Cats & $\%$ Pos. $(>23)^{*}$ & \% Pos. $(>15)^{*}$ & \% Pos. (Ave) \\
\hline 10 & Control & $\mathrm{SC} / \mathrm{SC}$ & 42 & 9 & 10.8 & 13.9 & 12.4 \\
\hline 10 & $\mathrm{FCV} \mathrm{F9}+\mathrm{FHV}+\mathrm{FPV}+\mathrm{FCp}$ & SC/SC & 42 & 9 & 38.5 & 42.3 & 40.4 \\
\hline 10 & $\mathrm{FCV} \mathrm{F9}+\mathrm{FHV}+\mathrm{FPV}+\mathrm{FCp}$ & SC/Oral & 42 & 10 & 39.2 & 42.7 & 41.0 \\
\hline
\end{tabular}

${ }^{*}$ Neutralization titer of $\geq 23$ as cut-off value; or neutralization titer of $\geq 15$ as cut-off value.

not correlate well for either FHV or FCp. The antibody titers we obtained were too low for us to make any meaningful conclusions. In vivo challenge data will be needed to assess protection for these antigens.

\section{Discussion}

Oral vaccination has been long sought after due to its convenient nature. Especially for cats, the concerns for fibrosarcoma formation as a result of needle injection, make oral vaccines a very attractive alternative. Here we provide data to show that experimental 4-way vaccine (FCV F9-FHV-FPV-FCp), when given SC followed by Oral administration (SC/Oral), decreased mortality caused by vs FCV-33585 to $10 \%$ while $44 \%$ mortality was observed when the same vaccine was given SC/SC. The unvaccinated cats had 78\% mortality against vs FCV33585 challenge. The median clinical scores were improved significantly as well. Furthermore, we did not observe any vaccination associated safety concerns post both vaccinations and prior to challenge for both SC/SC and SC/Oral groups. The results suggest that not only oral vaccination could be used for convenience and reducing the concerns of needle injection, it may also enhance the efficacy of the FCV vaccine.

The FCV serum cross-neutralization profile for the 4-way vaccine (FCV F9-FHV-FPV-FCp) was $40.4 \%$ for $\mathrm{SC} / \mathrm{SC}$ ) and $41 \%$ for SC/Oral. Whereas our previous observation was that cat serum generated by 1-dose oronasal route of administration had broader serum cross-neutralization profile than the 1-dose SC administration (Table 1 \& Ref. [14]). This discrepancy seems to suggest that the route of administration of the first dose for FCV vaccine is very critical in determining the FCV serum cross-neutralization. The second oral dose did not seem to affect the FCV serum cross-neutralization in comparison with SC route, even though it is clearly important for enhancing vaccine efficacy against vs FCV challenge. Also possible, the oral route was used in this FCV efficacy study (Tables 2-4), while oronasal route was used in the previous study (Table 1 \& Ref. [14]). The ability to 
Table 4. SN Titer analysis (geomean) of FHV (post-challenge serum), FPV and Chlamydia (FCp) (pre-challenge serum).

\begin{tabular}{cccccc}
\hline Treatment & Route & \# Cats & FHV & FPV & FCp \\
\hline Control & n/a & 9 & ND & ND & ND \\
FCV F9 + FHV + FPV + FCp & SC/SC & 9 & 2.3 & 36781 & 2 \\
FCV F9 + FHV + FPV + FCp & SC/Oral & 10 & 1.3 & 30574 & 4 \\
\hline
\end{tabular}

enhance serum cross-neutralization of FCV F9 by oronasal route might not be applicable to the oral route, despite the fact the SC/Oral is clearly more efficacious than SC/SC for FCV vaccine. Finally, 4-way vaccine (FCV F9-FHV-FPV-FCp) was used in this study, while only FCV F9 was used previously by oronasal route. The other antigen components in the 4-way vaccine might influence the FCV serum cross-neutralization profile.

The high FPV titers observed for groups of both SC/SC \& SC/Oral administration suggest good FPV efficacy could be expected for both groups. However, the low FHV and FCp titer, although not unexpected, did not provide much insight on the potential efficacy. The challenge studies will need to be conducted to determine the efficacies for FHV \& FCp.

\section{Acknowledgements}

The authors wish to thank Dr. Ed Dubovi at Cornell Diagnostic Lab (Ithaca, New York) for providing FCV strains 94580, 100869-1, 33585, 88287, 89391; Stan Berth for preparing lyophilized experimental vaccines.

\section{References}

[1] Thiel, H.J. and Konig, M. (1999) Caliciviruses: An Overview. Vet Microbiology, 69, 55-62. http://dx.doi.org/10.1016/S0378-1135(99)00088-7

[2] Gaskell, R.M., Radford, A.D. and Dawson, S. (2004) Feline Infectious Respiratory Disease. In: Chandler, E.A., Gaskell, C.J. and Gaskell, R.M., Eds., Feline Medicine and Therapeutics, 3rd Edition, Blackwell Publishing, Oxford, 577-595.

[3] Stuart, A.D. and Brown, T.D. (2006) Entry of Feline Calicivirus Is Dependent on Clathrin-Mediated Endocytosis and Acicification in Endosomes. Journal of Virology, 80, 7500-7509. http://dx.doi.org/10.1128/JVI.02452-05

[4] Makino, A., Shimojima, M., Miyazawa, T., Kato, K., Tohya, Y. and Akashi, H. (2006) Junctional Adhesion Molecule 1 Is a Functional Receptor for Feline Calicivirus. Journal of Virology, 80, 4482-4490. http://dx.doi.org/10.1128/JVI.80.9.4482-4490.2006

[5] Pesavento, P.A., Stokol, T., Liu, H., van der Lit, D.A., Gaffney, P.M. and Parker, J.S. (2010) Distribution of the Feline Calicivirus Receptor Junctional Adhesion Molecule A in Feline Tissues. Veterinary Pathology, 48, 361-368. http://dx.doi.org/10.1177/0300985810375245

[6] Pedersen, N.C., Elliott, J.B., Glasgow, A., Poland, A. and Keel, K. (2000) An Isolated Epizootic of Hemorrhagic-Like Fever in Cats Caused by a Novel and Highly Virulent Strain of Feline Calicivirus. Vet Microbiology, 73, 281-300. http://dx.doi.org/10.1016/S0378-1135(00)00183-8

[7] Radford, A.D., Dawsen, S., Gaskell, R.M., Foley, J., Hurley, K. and Pedersen, N.C. (2002) Haemorrhagic Fever, Oederma and High Mortality Associated with FCV Infection. The Vet Record, 151, 155.

[8] Schorr-Evans, E.M., Poland, A., Johnson, W.E. and Pedersen, N.C. (2003) An Epizootic of Highly Virulent Feline Calicivirus Disease in a Hospital Setting in New England. Journal of Feline Medicine and Surgery, 5, 217-226. http://dx.doi.org/10.1016/S1098-612X(03)00008-1

[9] Pesavento, P.A., MacLachlan, N.J., Dillard-Telm, L., Grant, C.K. andHurley, K.E. (2004) Pathologic, Immunohistochemical, and Electron Microscopic Findings in Naturally Occurring Virulent Systemic Feline Calicivirus Infection in Cats. Veterinary Pathology, 41, 257-263. http://dx.doi.org/10.1354/vp.41-3-257

[10] Hurley, K.F., Pesavento, P.A., Pedersen, N.C., Poland, A.M., Wilson, E. and Foley, J.E. (2004) An Outbreak of Virulent Systemic Feline Calicivirus Disease. JAVMA, 224, 241-249. http://dx.doi.org/10.2460/javma.2004.224.241

[11] Coyne, K.P., Jones, B.R.D., Kipar, A., Chantrey, J., Porter, C.J., Barber, P.J., Dawson, S., Gaskell, R.M. and Radford, A.D. (2006) Lethal Outbreak of Disease Associated with Feline Calicivirus Infection in Cats. Vet Record, 158, 544-550. http://dx.doi.org/10.1136/vr.158.16.544

[12] Abd-Eldaim, M., Potgieter, L. and Kennedy, M. (2005) Genetic Analysis of Feline Caliciviruses Associated with a 
Hemorrhagic-Like Disease. Journal of Veterinary Diagnostic Investigation, 17, 420-429. http://dx.doi.org/10.1177/104063870501700503

[13] Foley, J., Hurley, K., Pesavento, P.A., Poland, A. and Pedersen, N.C. (2006) Virulent Systemic Feline Calicivirus Infection: Local Cytokine Modulation and Contribution of Viral Mutant. Journal of Feline Medicine and Surgery, 8, 55-61. http://dx.doi.org/10.1016/j.jfms.2005.08.002

[14] Rong, S., Slade, D., Floyd-Hawkins, K. and Wheeler, D. (2006) Characterization of a Highly Virulent Feline Calicivirus and Attenuation of This Virus. Virus Research, 122, 95-108. http://dx.doi.org/10.1016/j.virusres.2006.07.003

[15] Reynolds, B.S., Poulet, H., Pingret, J.-L., Jas, D., Brunet, S., Lemeter, C., Etievant, M. and Boucraut-Baralon, C. (2009) A Nosocomial Outbreak of Feline Calicivirus Associated Virulent Systemic Disease in France. Journal of Feline Medicine and Surgery, 11, 633-644. http://dx.doi.org/10.1016/j.jfms.2008.12.005

[16] Meyer, A., Kershaw, O. and Klopfleisch, R. (2011) Feline Calicivirus Associated Virulent Systemic Disease: Not Necessarily a Local Epizootic Problem. Veterinary Record, 168, 589a. http://dx.doi.org/10.1136/vr.d160

[17] Schneider, K. and Truyen, U. (1998) Antigenic Variation among Feline Caliciviruses. Revue de Médecine Vétérinaire, 149, 1007-1011.

[18] Lauritzen, A., Jarrett, O. and Sabara, M. (1997) Serological Analysis of Feline Calicivirus Isolates from the United States and United Kingdom. Vet Microbiology, 56, 55-63. http://dx.doi.org/10.1016/S0378-1135(96)01252-7

[19] Radford, A.D., Dawson, S., Wharmby, C., Ryvar, R. and Gaskell, R.M. (2000) Comparison of Serological and Sequence-Based Methods for Typing Feline Calicivirus Isolates from Vaccine Failures. The Vet Record, 146, 117-123. http://dx.doi.org/10.1136/vr.146.5.117

[20] Radford, A.D., Dawson, S., Coyne, K.P., Porter, C.J. and Gaskell, R.M. (2006) The Challenges for the Next Generation of Feline Calicivirus Vaccines. Veterinary Microbiology, 117, 14-18. http://dx.doi.org/10.1016/j.vetmic.2006.04.004

[21] Baulch-Brown, C., Love, D.N. and Meanger, J. (1997) Feline Calicivirus: A Need for Vaccine Modification? Australian Veterinary Journal, 75, 209-213. http://dx.doi.org/10.1111/j.1751-0813.1997.tb10068.x

[22] Dawson, S., McArdel, F., Bennett, D., Carter, S.D., Bennett, M., Ryvar, R. and Gaskell, R.M. (1993) Investigation of Vaccine Reactions and Breakdowns after Feline Calicivirus Vaccination. The Vet Record, 132, 346-350. http://dx.doi.org/10.1136/vr.132.14.346 